\title{
Further Pathologies in Algebraic Geometry
}

\section{Citation}

Mumford, David B. 1962. Further pathologies in algebraic geometry. American Journal of Mathematics 84(4): 642-648.

\section{Published Version}

doi: $10.2307 / 2372870$

\section{Permanent link}

http://nrs.harvard.edu/urn-3:HUL.InstRepos:3446006

\section{Terms of Use}

This article was downloaded from Harvard University's DASH repository, and is made available under the terms and conditions applicable to Other Posted Material, as set forth at http:// nrs.harvard.edu/urn-3:HUL.InstRepos:dash.current.terms-of-use\#LAA

\section{Share Your Story}

The Harvard community has made this article openly available.

Please share how this access benefits you. Submit a story.

Accessibility 


\title{
Further Pathologies in Algebraic Geometry
}

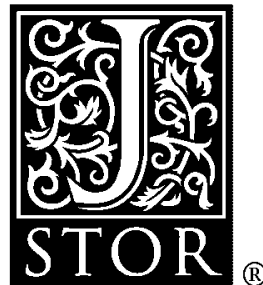

\author{
David Mumford
}

American Journal of Mathematics, Vol. 84, No. 4 (Oct., 1962), 642-648.

Stable URL:

http://links.jstor.org/sici?sici=0002-9327\%28196210\%2984\%3A4\%3C642\%3AFPIAG\%3E2.0.CO\%3B2-1

American Journal of Mathematics is currently published by The Johns Hopkins University Press.

Your use of the JSTOR archive indicates your acceptance of JSTOR's Terms and Conditions of Use, available at http://www.jstor.org/about/terms.html. JSTOR's Terms and Conditions of Use provides, in part, that unless you have obtained prior permission, you may not download an entire issue of a journal or multiple copies of articles, and you may use content in the JSTOR archive only for your personal, non-commercial use.

Please contact the publisher regarding any further use of this work. Publisher contact information may be obtained at http://www.jstor.org/journals/jhup.html.

Each copy of any part of a JSTOR transmission must contain the same copyright notice that appears on the screen or printed page of such transmission.

JSTOR is an independent not-for-profit organization dedicated to creating and preserving a digital archive of scholarly journals. For more information regarding JSTOR, please contact support@jstor.org. 


\title{
FURTHER PATHOLOGIES IN ALGEBRAIC GEOMETRY.*1
}

\author{
By David Mumford.
}

The following note is not strictly a continuation of our previous note [1]. However, we wish to present two more examples of algebro-geometric phenomena which seem to us rather startling. The first relates to characteristic $p$ behaviour, and the second relates to the hypothesis of the completeness of the characteristic linear system of a maximal algebraic family. We will use the same notations as in [1].

\section{I.}

The first example is an illustration of a general principle that might be said to be indicated by many of the pathologies of characteristic $p$ :

A non-singular characteristic $p$ variety is analogous to a general nonKähler complex manifold; in particular, a projective embedding of such a variety is not as "strong" as a Kähler metric on a complex manifold; and the Hodge-Lefschetz-Dolbeault theorems on sheaf cohomology break down in every possible way.

In this case we wish to look at the two dimensional cohomology of an algebraic surface $F$, non-singular, and of any characteristic but 0 . The surface we shall choose will (a) be specialization of a characteristic 0 surface $F^{\prime \prime}$, and (b) will satisfy $q=h^{0,1}=h^{1,0}$. Consequentily the second Betti number $B_{2}$ is the same, whether defined (i) as that of $F^{\prime}$ in the topological sense, (ii) as $h^{2,0}+h^{1,1}+h^{0,2}$, or (iii) following Igusa [2], as $\operatorname{Deg}\left(c_{2}\right)+4 q-2$. Let $\rho$ be the base number of $F$. Igusa showed that, in fact, $B_{2} \geqq \rho$. However, in characteristic 0 , one has the stronger result, $B_{2}=h^{2,0}+h^{1,1}+h^{0,2} \geqq 2 p_{g}+\rho$ (where $p_{g}=h^{2,0}=h^{0,2}$ is the geometric genus of $F$ ) as a result of the HodgeDolbeault theorems. Therefore the question arises whether this stronger inequality is valid in characteristic $p$. The answer is no.

A rather complicated example was discovered in 1961 by $\mathrm{J}$. Tate and A. Ogg. Here is a very simple example: let $E$ be a super-singular elliptic

* Received July 30, 1962.

${ }^{1}$ This work was supported by the Army Research Office (Durham). 
curve of characteristic $p$ (i.e. such that the rank of $\operatorname{End}(E)$ is 4 ). Let $F=E \times E$. In this case, in fact:

$$
\rho=B_{2}=6 ; \quad p_{g}=1
$$

Here $p_{g}=1$ since the sheaf $\Omega_{F}{ }^{2} \cong \mathfrak{O}_{F}$; and $B_{2}=6$ by Igusa's definition, for example, since $\operatorname{Deg}\left(c_{2}\right)=0$, and $q=2$. Finally $\rho=6$ since in general, for any two elliptic curves $E_{1}$ and $E_{2}$, one knows that the base number $\rho$ for $E_{1} \times E_{2}$ equals 2 plus the rank of $\operatorname{Hom}\left(E_{1}, E_{2}\right)$.

There remains one outstanding conjecture still neither proven nor disproven in characteristic $p$, which according to the general principle mentioned above ought to be false. This is the Regularity of the Adjoint, which may be stated as follows: if $V$ is a non-singular projective surface and if $H$ is a nonsingular hyperplane section, then

$$
H^{1}\left(\mathfrak{O}_{V}\right) \rightarrow H^{1}\left(\mathfrak{O}_{H}\right)
$$

is injective.

II.

The second example concerns space curves in characteristic 0 . Let $A$ be any family of non-singular space curves, and let $a \in A$ represent the curve $\gamma \subset P_{3}$. Let $T_{a}$ denote the Zariski tangent space to $A$ at $a$, and let $N$ denote the sheaf of sections of the normal bundle to $\gamma$ in $P_{3}$. Then it is well-known [3] that there is a "characteristic" map:

$$
T_{a} \rightarrow H^{0}(N)
$$

The problem of completeness consists in asking when, for given $\gamma$, there is a family $A$ containing $\gamma$ such that the characteristic map is surjective. Kodaira [3] has shown that such a family exists if $H^{1}(N)=(0)$. Our example shows that if $H^{1}(N) \neq(0)$, then there need not be such a family.

In fact, in our example, this incompleteness holds for every curve in an open set of the corresponding Chow variety. Consequently, it is also an example where the Hilbert scheme [4] has a multiple component, i. e. is not reduced at one of its generic points. Another corollary of this example is obtained by blowing up such a space curve $\gamma \subset P_{3}$ to a surface $E$ in a new three-dimensional variety $V_{3}$. Then Kodaira [5] has shown essentially that the local moduli scheme of the variety $V_{3}$ is isomorphic to the germ of the 
Hilbert scheme of $P_{3}$ at the point corresponding to $\gamma$. Therefore we have constructed a non-singular projective three-dimensional variety whose local moduli scheme is nowhere reduced; in other words, any small deformation of $V_{3}$ is a variety the number of whose moduli is less than the dimension of $H^{1}(\Theta)$ (where $\Theta$ is the sheaf of vector fields).

The curves $\gamma$ that we have in mind have degree 14 and genus 24 . In the following, $h$ will stand for the divisor class on $\gamma$ induced by plane sections, and $K_{\gamma}$ will stand for the canonical divisor on $\gamma$; also $F$ will stand for a cubic or quartic surface in $P_{3}$, and $H$ will stand for the (Cartier) divisor class on $F$ induced by plane sections. The first step is partial classification of all space curves of this degree and genus, which confirms the results of $M$. Noether's well-known table [6].

(A) Any non-singular space curve $\gamma$ of degree 14 and genus 24 is contained in a pencil $P$ of quartic surfaces.

Proof. Since $\operatorname{Deg}(4 h)=56$, and $\operatorname{Deg}\left(K_{\gamma}\right)=46$, the linear system $|4 h|_{\gamma}$ is non-special, ${ }^{2}$ and has dimension $56-24=32$. Since there is a $34-$ dimensional family of quartics in $P_{3}$, (A) follows.

There are 2 cases: (a) the pencil has no fixed components, and (b) the pencil has fixed components. In case (a), note first that if $F^{\prime \prime}$ and $F^{\prime \prime}$ span $P$, then $F^{\prime} \cdot F^{\prime \prime}=\gamma+c$, where $c$ is a conic. Now $c$ has at most double points, hence $\gamma+c$ has at most triple points. Therefore no point $x$ is a double point for both $F^{\prime}$ and $F^{\prime \prime}$. Noting that both $F^{\prime}$ and $F^{\prime \prime}$ are non-singular and transversal along $\gamma-c$, hence at all but a finite number of points of $\gamma$, it follows that almost every $F \in P$ is non-singular everywhere along $\gamma$.

(B) Every algebraic family of space curves of type (a) has dimension less than or equal 56.

Proof. It is enough to show that every family of pairs $(\gamma, F)$ consisting of such curves $\gamma$, and quartics $F \supset \gamma, F$ being non-singular along $\gamma$, has dimension at most 5\%. Now since all such quartics contains conics, they are not generic [7], and there is at most a $34-1=33$ dimensional family of quartics $F$ involved in such a family of pairs. Moreover, the dimension of the set of all $\gamma$ on one such $F$ can be computed from the Riemann-Roch theorem on $F$ :

\footnotetext{
${ }^{2}$ Here and below, $|D \cdot|_{v}$ always means the linear system on $V$ in which the Cartier divisor $D$ varies. Also, $\left(D^{2}\right)_{V}$ always denotes the self-intersection of $D$, as a divisor class on $D$ (assuming $D$ effective).
} 


$$
\operatorname{dim}|\gamma|_{F}=\frac{\operatorname{Deg}\left(\gamma^{2}\right)_{F}}{2}+1+\left\{\operatorname{dim} H^{1}\left(\boldsymbol{O}_{F}(\gamma)\right)-\operatorname{dim} H^{2}\left(\boldsymbol{O}_{F}(\gamma)\right)\right\}
$$

But $\left(\gamma^{2}\right)_{F} \equiv K_{\gamma}$ on $\gamma$, hence $\operatorname{Deg}\left(\gamma^{2}\right)_{F}=46$. Moreover, $H^{i}\left(\mathscr{O}_{F}(\gamma)\right)$ is dual to $H^{2-i}\left(\mathscr{O}_{F}(-\gamma)\right)$ by Serre duality. This cohomology group can be computed from the exact sequence:

$$
0 \rightarrow \mathbb{O}_{F^{r}}(-\gamma) \rightarrow \mathbb{O}_{F^{\prime}} \rightarrow \mathbb{O}_{\gamma} \rightarrow 0 .
$$

It follows that both are zero, hence $\operatorname{dim}|\gamma|_{F}=24$. Therefore, indeed, the set of pairs $\left(\gamma, F^{\prime}\right)$ has dimension at most $33+24=5 \% .^{3}$

Now consider case (b). Such a $\gamma$ must be contained in a reducible quartic, hence in a plane, a quadric, or a cubic surface. The first two possibilities are readily checked and it happens that they contain no curves of the required degree and genus. Moreover, such a curve is contained in a unique cubic surface $F$, because $\operatorname{Deg}(\gamma)=14>9=\operatorname{Deg}\left(F^{\prime} \cdot F^{\prime \prime}\right)$, for two distinct cubic surfaces $F^{\prime}$ and $F^{\prime \prime}$. We will say that $\gamma$ is of type $\left(b_{0}\right)$ if the cubic $F$ is nonsingular; otherwise, we will say that $\gamma$ is of type $\left(b_{1}\right)$.

(C) Every maximal algebraic family of curves $\gamma$ of type $\left(b_{0}\right)$ has dimension 56 .

Proof. Let $\gamma$ be a curve of type $\left(b_{0}\right)$, and let $F$ be the corresponding cubic surface. Since $K_{F} \equiv-H$, by the Riemann-Roch theorem on $F$ :

$$
\operatorname{dim}|\gamma|_{F^{\prime}}=\frac{\operatorname{Deg}(\gamma \cdot \gamma+H)_{F}}{2}+\left\{\operatorname{dim} H^{1}\left(\boldsymbol{O}_{F^{F}}(\gamma)\right)-\operatorname{dim} H^{2}\left(\boldsymbol{O}_{F}(\gamma)\right)\right\} .
$$

But $K_{\gamma} \equiv \gamma \cdot\left(\gamma+K_{F}\right)$, hence $46=\operatorname{Deg}\left(\gamma^{2}\right)_{F}-\operatorname{Deg}(\gamma \cdot H)_{F}=\operatorname{Deg}\left(\gamma^{2}\right)_{F}-14$, hence $\operatorname{Deg}\left(\gamma^{2}\right)_{F}=60$. Also, $H^{i}\left(\mathscr{O}_{F}(\gamma)\right)$ is dual to $H^{2-i}\left(\widehat{O}_{F}(-H-\gamma)\right)$, and this group can be computed from the exact sequence:

$$
0 \rightarrow \mathfrak{O}_{F}(-H-\gamma) \rightarrow \mathfrak{O}_{F} \rightarrow \mathfrak{O}_{(H+\gamma)} \rightarrow 0 .
$$

Since $H+\gamma$ is a reduced and connected curve, $H^{0}\left(\mathscr{O}_{H+\gamma}\right)=k$ (constants), and this implies $H^{i}\left(\mathscr{O}_{F}(-H-\gamma)\right)=(0)$ for $i=1$ and 2. Putting all this together, we see that $\operatorname{dim}|\gamma|_{F}=3 \%$. Since there is a 19-dimensional family

${ }^{3}$ It may be objected that we have used the Riemann-Roch theorem, and Serre duality as though $F$ were non-singular. But since $F$ is non-singular along $\gamma$, the former can be proved by means of the exact sequence:

$$
0 \rightarrow \mathcal{O}_{F} \rightarrow \mathcal{O}_{F^{*}}(\gamma) \rightarrow \mathcal{O}_{\gamma}\left(\left(\gamma^{2}\right)_{F}\right) \rightarrow 0 .
$$

And the latter can be proven either (a) directly by resolving the singularities of $F$ and comparing the cohomology on $F$ and on its resolution, or (b) as a consequence of Grothendieck's general theory [8]. In the second case, one merely has to note that $F^{\prime}$ is always a Cohen-Macauley variety; and since it is a quartic surface the canonical sheaf is simply $\mathcal{O}_{F}$ itself. 
of cubic surfaces, (C) follows if we show that a generic $\gamma$ in a maximal algebraic family is contained in a generic cubic surface. But let $\gamma \subset F$ be any curve of the family. Then recalling that the divisor class group of any nonsingular cubic surface is the same as that of any other, it follows that if the set of all non-singular cubic surfaces are suitably parametrized the invertible sheaf $\mathscr{O}_{F}(\gamma)$ will be a specialization of an invertible sheaf $L$ defined on the generic cubic surface $F^{*}$. And since $H^{i}\left(\mathscr{O}_{F}(\gamma)\right)=(0)$ for $i=1$ and 2 , by the upper semi-continuity of cohomology [9], we conclude that $H^{i}(L)=(0)$ for $i=1$ and 2 , and that all sections of $\mathscr{O}_{F}(\gamma)$ are specializations of sections of $L$. Therefore $\operatorname{dim} H^{0}(L)=38$; and since almost all sections of $\mathfrak{O}_{F}(\gamma)$ are non-singular, so are almost sections of $L$. Hence there is a non-singular $\gamma^{*} \subset F^{*}$ specializing to $\gamma \subset F$. QED.

Now suppose $C$ is the Chow variety of non-singular curves of degree 14, and genus 24. Let $C_{b} \subset C$ be the locus of curves of type $(b)$, and let $C_{b_{1}} \subset C_{b}$ be the locus of curves of type $\left(b_{1}\right)$. Then it is clear that $C_{b}$ and $C_{b_{1}}$ are closed (possibly reducible) subvarieties of $C$. By (B) and $(\mathrm{C})$, every component of $C-C_{b}$ has dimension $\leqq 56$, and every component of $C_{b}-C_{b_{1}}$ has dimension $=56$. Therefore if $C_{0}$ equals $C$ minus $C_{b_{1}}$ and minus the closure of $C-C_{b}, C_{0}$ is an open set in the Chow variety, of dimension 56, and parametrizing almost all curves of type $\left(b_{0}\right)$.

We shall now single out a set of components of $C_{0}$ such that, if $N$ is the normal sheaf to a $\gamma$ in one of these components, then $\operatorname{dim} H^{0}(N)=5 \%$. In fact, we say that $\gamma \subset F$ is of type $\left(b^{\prime}{ }_{0}\right)$ if there is a line $E$ on $F$ such that $\gamma \equiv 4 H+2 E$ on $F$. Then the corresponding $C^{\prime}{ }_{0} \subset C_{0}$ which is the locus of such curves is clearly closed in $C_{0}$. But it is also open: if $\gamma^{*} \subset F^{*}$ specializes to $\gamma \subset F$, and if $\gamma \equiv 4 H+2 E$ on $F$, then first of all, there is a line $E^{*} \subset F^{*}$ (possibly only rationally defined after a suitable base extension) which specializes to $E$; and secondly, since the divisor class group is discrete and constant for all non-singular cubics,

$$
\gamma-4 H-2 E \equiv 0 \text { implies } \gamma^{*}-4 H^{*}-2 E^{*} \equiv 0 .
$$

Therefore $\gamma^{*}$ is of type $\left(b^{\prime}{ }_{0}\right)$.

(D) If $\gamma \subset F$ is of type $\left(b^{\prime}{ }_{0}\right)$, then $\operatorname{dim} H^{0}(N)=5 \%$.

Proof. Let $N_{F}$ be the sheaf of normal vector fields to $\gamma$ and in $F$, and let $N_{P}$ be the sheaf of normal vector fields to $F$, and in $P_{3}$, which are defined along $\gamma$. Then we have the sequence:

$$
0 \rightarrow N_{F} \rightarrow N \rightarrow N_{P} \rightarrow 0 .
$$


But if $D$ is a non-singular divisor on a non-singular variety $V$, then its normal sheaf is isomorphic to $\mathscr{O}_{D}\left(\left(D^{2}\right)_{V}\right)$. Therefore $N_{F} \cong \mathscr{O}_{\gamma}\left(\left(\gamma^{2}\right)_{F}\right)$ and $N_{F} \cong \mathscr{O}_{\gamma}(3 h)$. But since $K_{\gamma} \equiv\left(\gamma^{2}\right)_{F}+\gamma \cdot K_{F} \equiv\left(\gamma^{2}\right)_{F}-h$, it follows that $\left(\gamma^{2}\right)_{F}$ is a non-special divisor, of degree 60 in fact. Therefore $H^{1}\left(N_{F}\right)=(0)$ and $\operatorname{dim} H^{0}\left(N_{F}\right)=60-(24-1)=3 \%$. On the other hand, by the RiemannRoch theorem for curves,

$$
\begin{aligned}
\operatorname{dim} H^{0}\left(\mathfrak{O}_{\gamma}(3 h)\right) & =42-(24-1)+\operatorname{dim} H^{0}\left(\mathbb{0}_{\gamma}\left(K_{\gamma}-3 h\right)\right) \\
& =19+\operatorname{dim} H^{0}\left(\mathbb{0}_{\gamma}\left(\left(\gamma^{2}\right)_{F}-4 h\right)\right) \\
& =19+\operatorname{dim} H^{0}\left(\mathbb{0}_{\gamma}(2 \gamma \cdot E)\right)
\end{aligned}
$$

(using the hypothesis $\gamma \equiv 4 H+2 E$ ). But now, use the exact sequence:

$$
0 \rightarrow \mathbb{O}_{F}(-4 H) \rightarrow \mathscr{O}_{F}(2 E) \rightarrow \mathscr{O}_{\gamma}(2 \gamma \cdot E) \rightarrow 0 .
$$

It is readily seen that $H^{i}\left(\mathbb{O}_{F}(-4 H)\right)=(0)$ for $i=0$ and 1 , and that $\operatorname{dim} H^{0}\left(\boldsymbol{O}_{F}(2 E)\right)=1$. Putting all this information together we conclude: $\operatorname{dim} H^{0}(N)=3 \%+19+1=5 \%$ QED.

It remains only to note:

(E) If $F$ is any non-singular cubic surface, and $E \subset F$ is any line, there exist non-singular curves $\gamma \in|4 H+2 E|$, and they have degree 14 and genus 24.

Proof. The degree and genus of such a $\gamma$ are computed by the usual formulae, recalling that $\operatorname{Deg}\left(E^{2}\right)_{F}=-1$. To see that such a $\gamma$ exists, it suffices, by the characteristic 0 Bertini theorem, to prove that $|4 H+2 E|$ has no base points. But the only possible base points are the points of $E$, and we use the exact sequence:

$$
0 \rightarrow \mathfrak{O}_{F}(4 H+E) \rightarrow \mathfrak{O}_{F}(4 H+2 E) \rightarrow \mathscr{O}_{E}(2) \rightarrow 0 .
$$

Since the sections of $\mathscr{O}_{E}(2)$ have no base points, it suffices to prove $H^{1}\left(\mathfrak{O}_{r}(4 H+E)\right)=(0)$. But this follows from the sequence:

$$
0 \rightarrow \mathfrak{O}_{F}(4 H) \rightarrow \mathfrak{O}_{F}(4 H+E) \rightarrow \mathfrak{O}_{E}(3) \rightarrow 0,
$$

since $H^{1}\left(\mathscr{O}_{F}(4 H)\right)=(0)$, and $H^{1}\left(\mathscr{O}_{E}(3)\right)=(0)$. QED. 


\section{REFERENCES.}

[1] D. Mumford, "Pathologies of modular algebraic surfaces," American Journal of Mathematics, vol. 83 (1961), p. 339.

[2] J. I. Igusa, "Betti and Picard numbers of abstract algebraic surfaces," Proceedings of the National Academy of Sciences, vol. 46 (1960), p. 724.

[3] K. Kodaira, "A theorem of completeness of characteristic systems for analytic families of compact submanifolds of complex manifolds," Annals of Mathematics, vol. 72 (1962), p. 146.

[4] A. Grothendieck, "Techniques de construction et théorèmes d'existence en géométrie algébrique, IV: Les schémas de Hilbert," Séminaire Bourbaki, Exposé 221, 1961.

[5] K. Kodaira, "On stability of compact submanifolds of complex manifolds," to appear in American Journal of Mathematics.

[6] M. Noether, Zur Grundlegung der Theorie der Algebraischen Raumkurven, Steiner Preisschrift, Berlin, 1882.

[7] S. Lefschetz, "On certain numerical invariants of algebraic varieties with applications to Abelian varieties," (Memoire Bordin), Transactions of the American Mathematical Society, vol. 22 (1921), p. 327.

[8] A. Grothendieck, "Théorèmes de dualité pour les faisceaux algebriques cohérents," Séminaire Bourbaki, Exposé 149, 1957.

[9] W. L. Chow and J. I. Igusa, "Cohomology theory of varieties over rings," Proceedings of the National Academy of Sciences, vol. 44 (1958), p. 1244. 